\title{
GENERATION OF TRANSPORT PATHS IN FRACTURED POROUS MEDIA
}

\author{
JAKUB ŘÍHA*, JiŘina KRÁlOVCOVÁ \\ The Institute for Nanomaterials, Advanced Technology and Innovation, Technical University of Liberec, \\ Studentská 1402/2, 46117 Liberec 1, Czech Republic \\ * corresponding author: jakub.riha@tul.cz
}

\begin{abstract}
In this article, a method for generation of transport paths in combined equivalent porous media / discrete fracture network computational meshes is proposed as an alternative to a particle tracking method. It is based on a computation of the functional of the velocity and concentration individually for each element of a mesh. Its functionality is demonstrated on two test cases.
\end{abstract}

KEYWORDS: transport paths; fractured porous media; Flow123D; particle tracking.

\section{INTRODUCTION}

This article deals with the issue of generation of transport paths in fractured porous media. Currently, the transport paths are perceived as a sequence of points in a space defining a path of, in a sense, dominant propagation of transported substance from a source through a simulation domain.Flow and transport paths and quantitative parameters along them could serve as suitable characteristics for an assessment of transport processes in a particular domain and/or for mutual comparison of different sites from the point of their migration capabilities, even in the cases when we have full concentration transport results available for an evaluation.

There are three basic variants of fractured porous media models:

(1.) equivalent porous media (EPM) [14;

(2.) discrete fracture network (DFN) [12, 14];

(3.) combination of EPM and DFN.

The combined approach implies the presence of elements of different dimensions in a mesh (elements representing the DFN are of a lower dimension than elements representing the EPM).

Usually, the transport paths are derived based on the steady flow simulation results. The commonly used method for their generation is the particle tracking [9]. Various implementations of this method can be found in existing literature [1, 2, 6, 10, 11, 18. This method uses a simulated velocity field for the computation of streamlines of particles transported purely by advection. A velocity field, as an output of a numerical method, cannot naturally be continuous (velocities are, based on the chosen numerical method, computed either in elements vertices or in their barycenters). It is inevitable to use some interpolation method for us to be able to describe the velocity field in an arbitrary point of a space-time. The insufficiency of the particle tracking method appears in the case of a combined EPM/DFN model. In such case, the method could prove, due to the necessary interpolation, unable to provide a transport path copying the discrete fractures (lower dimension subdomains) even though the lower dimension domain, or its parts, constitutes the prevalent transport direction. These difficulties are introduced, for example, in Willmann at al. (2013) [17, however, the solution proposed there is applicable only for regular, orthogonal meshes.

Since the particle tracking method is not suited for combined EPM/DFN models, an alternative method based on a computation of the functional of velocity and concentration individually for each element of a mesh is proposed in this article.

\section{Methods}

Further presented procedure for transport path assessment utilizes results of not only underground steady flow but also solute transport simulations. The procedure was originally invented for and tested on the base of Flow123d simulator [4, 5, 15] results. Flow123d is a software for a simulation of a water flow, reactive solute transport and heat transfer in a heterogeneous porous and fractured medium. In particular, it is suited for a simulation of underground processes in a granite rock massive. The simulator is able to explicitly describe processes in a 3D medium, 2D fractures and $1 \mathrm{D}$ channels as well as an exchange between domains of different dimensions.

We suppose the flow and transport processes are simulated on a domain $\Omega$, which reflects the fractured porous medium. A computational mesh is acquired by discretization of $\Omega$ using simplicial elements of different dimensions (lines, triangles and tetrahedra). Subsequently, the underground water flow is computed using this mesh. The computation of the steady flow is given by the Darcy equation and the continuity equation [7, 14, 16]. This task can be solved using, among others, the primary (PFEM) or mixed-hybrid (MHFEM) finite element method [3, 13]. The principal unknowns of the system are the pressure head 
and the Darcy flux either in element vertices (PFEM) or in their barycentres (MHFEM). The motion of substances dissolved in water is governed by the advection and the hydrodynamic dispersion. This task can be solved using either the Transport operator splitting method (TOS) 8, which represents an explicit in time finite volume solver, or the implicit in time discontinuous Galerkin method (DG) [8]. The principal unknown is the concentration of a substance either in element vertices (DG) or in their barycentres (TOS).

Altogether, for the purpose of a transport path determination, we suppose the following:

- It is given a domain $\Omega$ and discretization of the domain onto a set of elements $\left\{e_{i} \in \Omega_{e}: \bigcup_{i} e_{i}=\right.$ $\left.\Omega_{e}\right\}$ (mentioned also as a mesh or a computational mesh).

- We have computed the flow field over the domain $\Omega$; i.e. we have the value $v_{e_{i}}$ as the actual velocity on every element $e_{i}$ of the mesh $\Omega_{e}$. (The actual velocity is computed by dividing the Darcy velocity by porosity.)

- It is selected start point $P_{S}$ we need to determine the transport path from an inner point of domain $\Omega$; thereby we have selected the start element $e_{S}$ of $\Omega_{e}$.

- We have computed concentration field; i.e. we have the values $c_{e_{i}}$ on all the elements $e_{i}$ at a particular time $t$.

- The flow and transport fields were obtained under the parameters of the simulations consistent with the requirements of the subsequent evaluation (this clause might include, for example, the following demand: the transport has a source of solutes only in the initial point or starting element, from which we need to follow the transport path - i.e., the initial point of the required transport path); the time $t$, at which we have the concentration field, is long enough for the concentration field to be utilizable.

Our aim is to provide a sequence of points through the domain $\Omega$ that constitutes the dominant path of solutes transport from the initial point $P_{S}$. As it was already mentioned, the commonly used method for generation of transport paths is particle tracking [9, which is based on a known velocity field. In our case, we want to solve this task generally on a mesh with elements of various dimensions (combination of the EPM and DFN approach). Using the particle tracking method, the problems caused by the interpolation of a velocity into an arbitrary point of space-time arise 17. This may lead to a transport path, which is unable to copy the course of fractures (represented by lower dimension elements) even though the velocity in fractures may be a couple orders of magnitude higher than the velocity in the surrounding porous media.

Our intention was to propose a method for transport path generation that not only eliminates these problems but also provides more flexibility in a sense of different meanings of the generated transport path. The method should be applicable to irregular (e.g. tetrahedral) computational meshes.

Our method (further described below) originates in the description of advective transport where two principal quantities are the concentration of substance and flow velocity. The method is required to be able to find both high risk types of a transport path - the most abundant and the fastest one. The fastest path, which follows the highest flow velocity direction from a selected original point, is determinable based on the results of the flow simulation (velocity field). The most abundant one, which follows the direction of the lowest concentration gradient from a selected original point, is traceable based on the results of an advective transport simulation (concentration field). As an outcome of this analysis, we propose a method based on an evaluation of an empirical functional, involving both mentioned aspects, computed as a weighted sum of two quantities possibly on every element of the mesh. The first quantity reflects the influence of the flow field and the second one the influence of the concentration field. To avoid summing quantities whose values can be in different orders of magnitude, we use their relative (dimensionless) values.

We proposed an empirical discrete functional $f_{e}(v, c)$, computed individually for each element $e$ of a mesh, in a form:

$$
f_{e}(c, v)=\omega_{v} \frac{\left|v_{e}\right|}{|v|_{\max }}+\omega_{c} \frac{c_{e}}{c_{\max }},
$$

where $v[\mathrm{~m} / \mathrm{s}]$ is the actual velocity on the given element, $v_{\max }[\mathrm{m} / \mathrm{s}]$ is the highest actual velocity found in the simulated domain, $c[\mathrm{~kg} / \mathrm{m} 3]$ is the concentration of a substance on the given element and $c_{\max }$ $[\mathrm{kg} / \mathrm{m} 3]$ is the highest concentration found in the simulated domain. The weights $\omega_{v}$ and $\omega_{c}[-]$ form the linear combination of the flow and concentration terms. They are empirical constants providing a greater flexibility of the functional usage. The suitable choice of weights $\omega_{v}, \omega_{c}$ allows emphasizing of one of the following meanings of the subsequently generated transport path:

- the fastest path - the sequence of elements through which the transported substance reaches a boundary in the shortest time;

- the most abundant path - the sequence of elements through which the biggest amount of substance is transported to a boundary in the given time.

Based on the computed functional, the transport path is stepwise generated as a sequence of elements from the source to a boundary of the simulated area using the following algorithm:

(1.) Add the element $e_{S}$ representing a source (the initial element in which the start point $P_{S}$ is) into a transport path sequence.

(2.) Take the last (last added) element of transport path and label it $E_{A C T}$. 

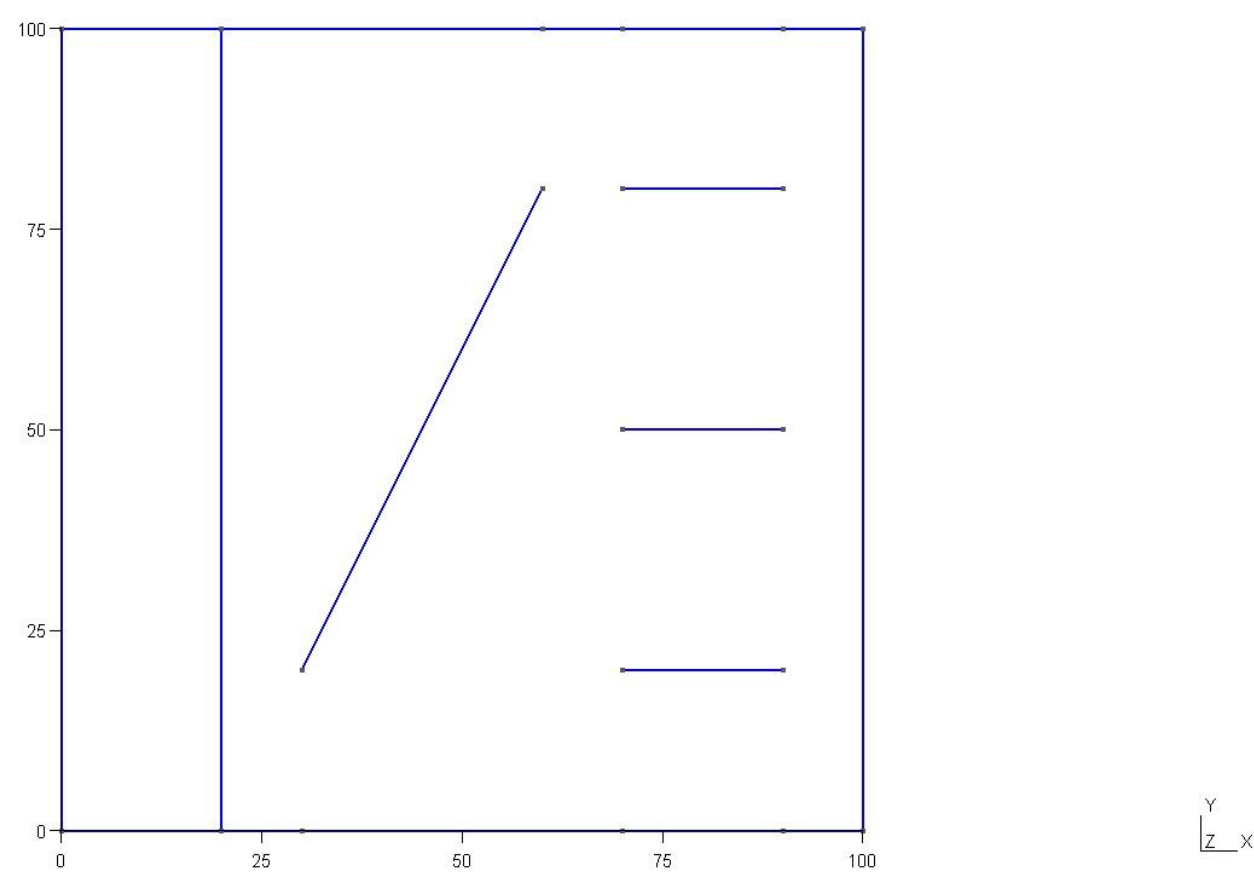

Figure 1. Test case A - geometry.

(3.) In case the element $E_{A C T}$ is on the domain boundary the algorithm is terminated.

(4.) Form a NEIGH_SET as a set of all such neighbouring elements of EACT not so far present in the generated transport path.

(5.) If the NEIGH_SET is null, the algorithm is terminated.

(6.) For every element $E_{N E I G H}$ from NEIGH_SET, compute the functional, if needed, and find the element $E_{M A X F}$ with the highest value of the functional.

(7.) Verify whether the flux from the element $E_{A C T}$ to the element $E_{M A X F}$ is positive (to prevent the path from cycling):

(a) If so, add the element $E_{M A X F}$ to the transport path sequence. Continue with the step (2).

(b) If not, remove the element $E_{M A X F}$ from the set NEIGH_SET. Continue with the step (5).

Using this procedure, we get a transport path as a sequence of selected mesh elements. Concentration values used in the functional are obtained from the simulation of a solute transport; i.e. a transient process, hence the generated transport path is, in general, dependent on the time we have the concentration values at.

The proposed procedure is designed for the computation of the functional based on outputs of the Flow123d simulation software [4, [5, 15].

\section{Examples, RESUlts AND DISCUSSION}

The proposed method and its aspects were tested on several $2 \mathrm{D}$ and $3 \mathrm{D}$ cases. In this part, the results for two $2 \mathrm{D}$ cases are shown and discussed.

\subsection{Test CASE A}

The geometry of the first test case is shown in Figure 1 . It is a $2 \mathrm{D}$ area of a size of $100 \times 100 \mathrm{~m}$ (in the $x y$ plane) with $1 \mathrm{D}$ fractures (their aperture is $0.01 \mathrm{~m}$ ). The hydraulic conductivity of fractures is two orders of magnitude higher than a hydraulic conductivity of rock. The porosity is constant in the whole area. The nature of flow is given by the prescribed Dirichlet boundary conditions of constant pressure head on boundaries $x=0 \mathrm{~m}(p=1 \mathrm{~m})$ and $x=100 \mathrm{~m}(p=$ $0 \mathrm{~m})$. The concentration source is situated in point $[10 ; 50]$.

The flow and the solute transport were computed. The simulation time for the transport was chosen so that the output concentration field is close to a stabilization. Based on the outputs of these simulations, the functional described above was computed and the transport path was generated for two different combinations of weights $\omega_{c}$ and $\omega_{v}$ :

- Case $\omega_{c}=0$ and $\omega_{v}=1$. The result is shown in Figure 2p. Only the velocity is considered. As expected, the transport path copies the fractures when possible. This is the fastest path.

- Case $\omega_{c}=1$ and $\omega_{v}=0$. The result is shown in Figure 2a. Only the concentration is considered. This is the most abundant path (in the given time). 

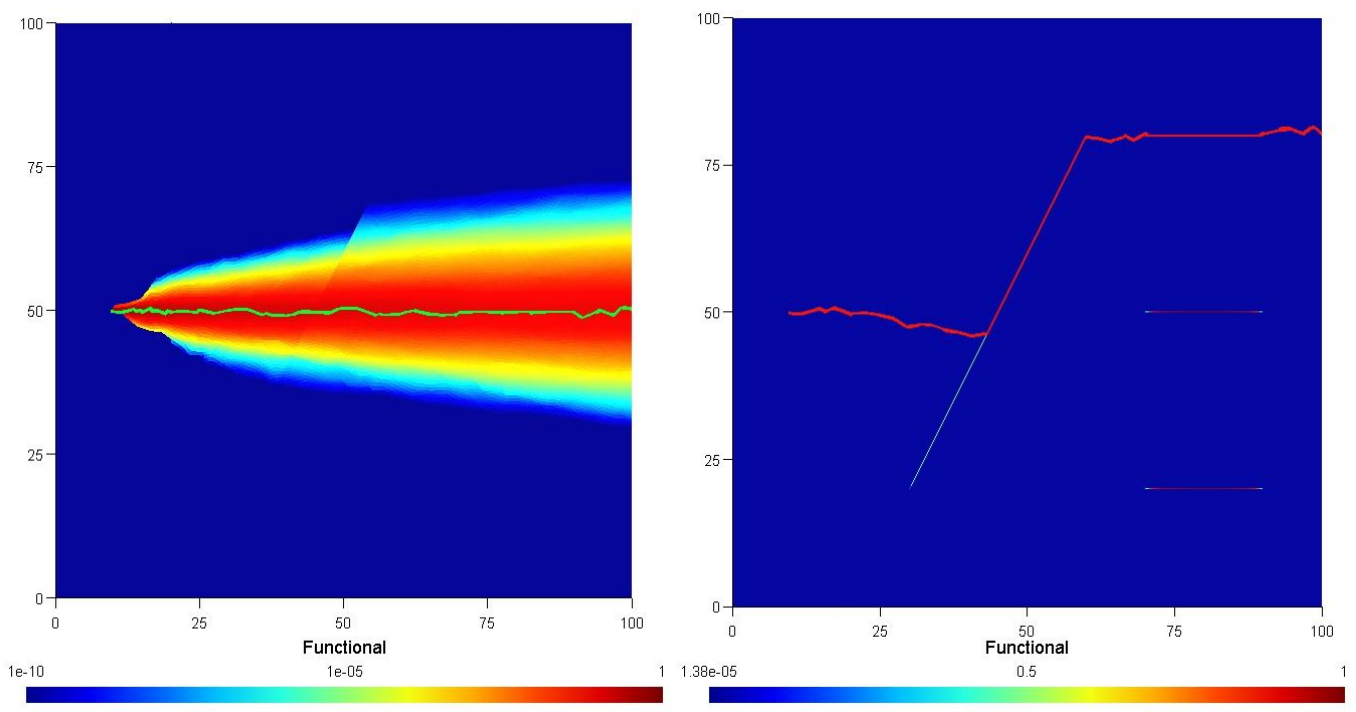

FiguRE 2. Test case A - transport paths: $\omega_{c}=1$ and $\omega_{v}=0$ (left); $\omega_{c}=0$ and $\omega_{v}=1$ (right). The background for the depicted transport paths is a value of the elementwise computed functional.

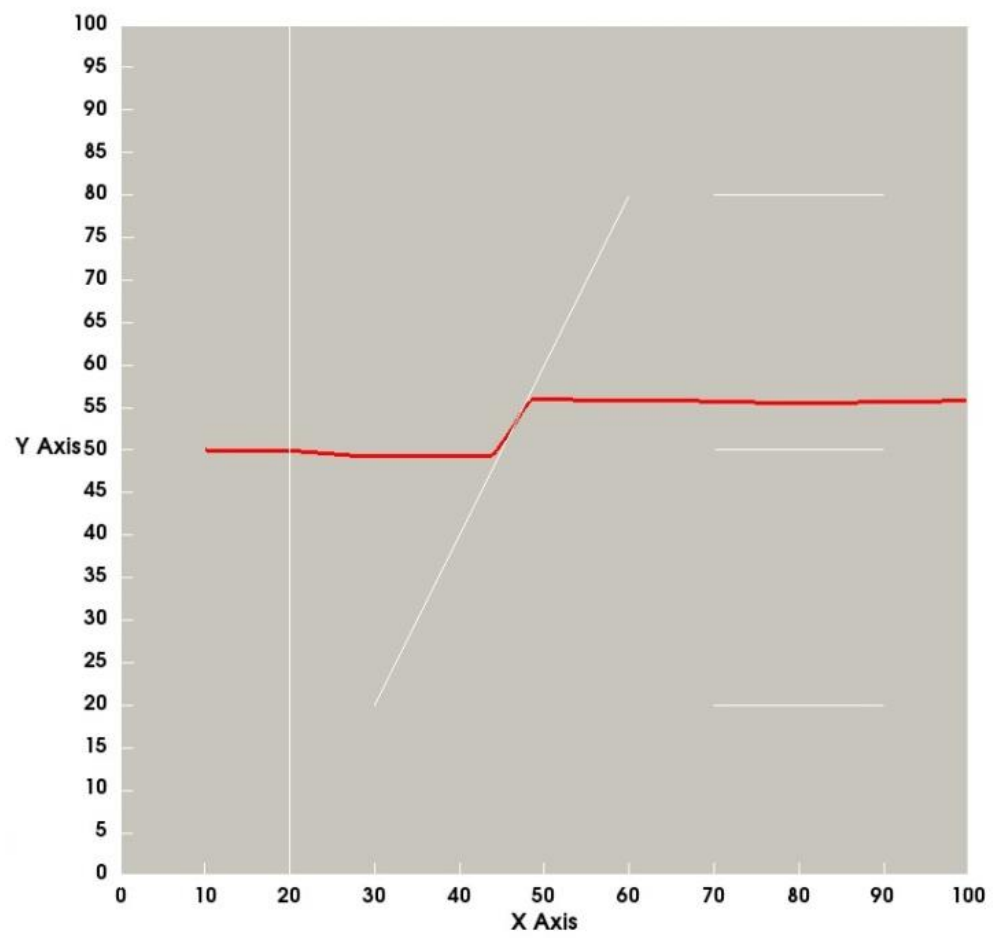

Figure 3. Test case A - particle tracking.

It is apparent, from Figure 2, that for different combinations of weights, we get different transport paths. Through the fastest path, the concentration reaches a boundary in the shortest time albeit in trace amount due to a dilution.

For a comparison, the transport path generated using the particle tracking method implemented in ParaView software [1, 2] is shown in Figure 3 This figure clearly demonstrates the disadvantage of this method described in the introduction. The gen- erated transport path intersects the fracture. It comes close to copying the fracture, but it never copies it entirely. This may cause a flawed assessment of the transport path and complicate its description (for example, from the viewpoint of the delay time or the rock types, the transport path crosses).

Other combination of weights than the ones shown here may also have a significance. In the interest of briefness, they are not discussed. 


\begin{tabular}{lcc}
\hline & Hydraulic conductivity $[\mathrm{m} / \mathrm{s}]$ & Porosity $[-]$ \\
\hline Fracture zones & $7 \cdot 10^{-7}$ & 0.1 \\
Low permeability rock & $7 \cdot 10^{-10}$ & 0.01 \\
High permeability rock & $7 \cdot 10^{-8}$ & 0.05 \\
\hline
\end{tabular}

TABle 1. Test case B - model parameters.

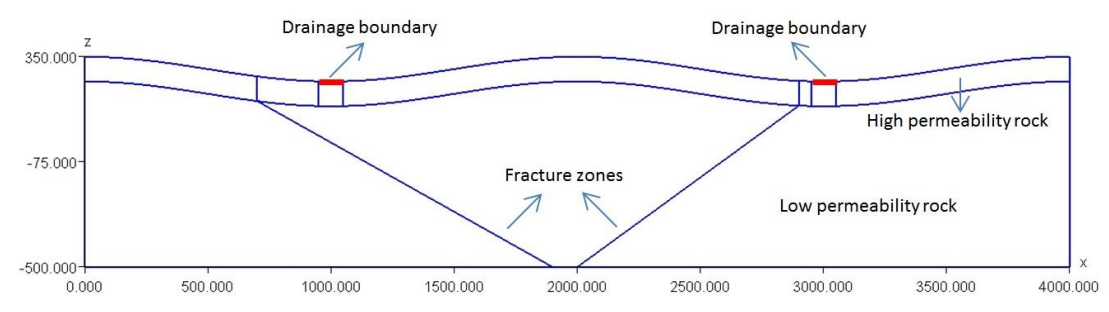

Figure 4. Test case B - geometry.

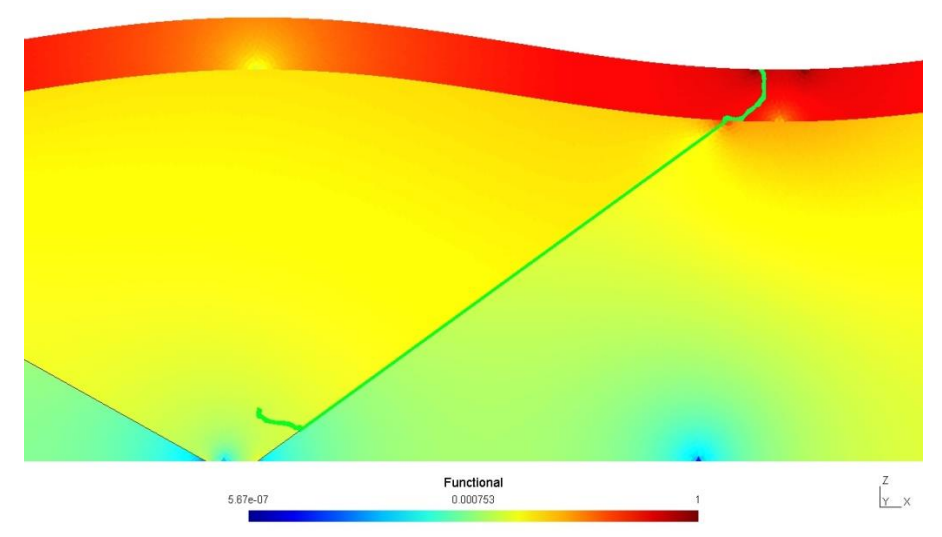

Figure 5. Test case B - transport path for $\omega_{c}=0$ a $\omega_{v}=1$. The background is a value of the elementwise computed functional.

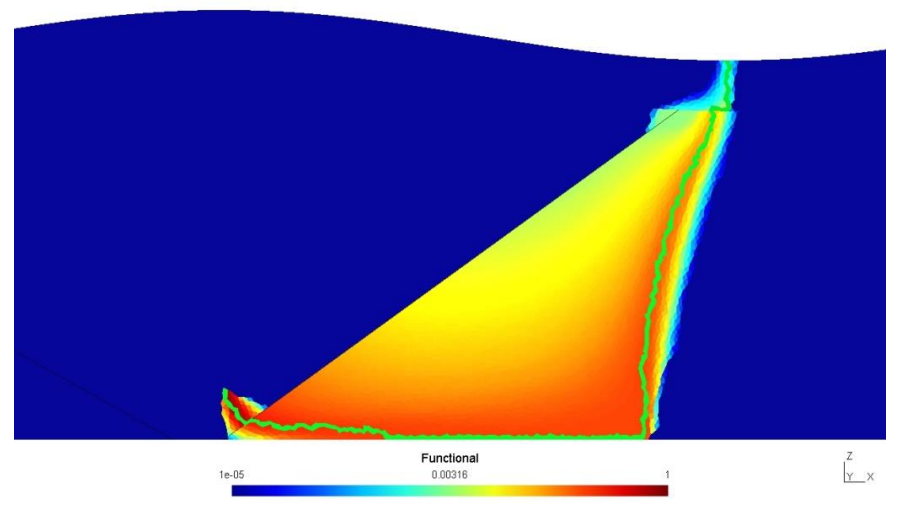

Figure 6 . Test case B - transport path for $\omega_{c}=1$ a $\omega_{v}=0$. The background is a value of the elementwise computed functional. 


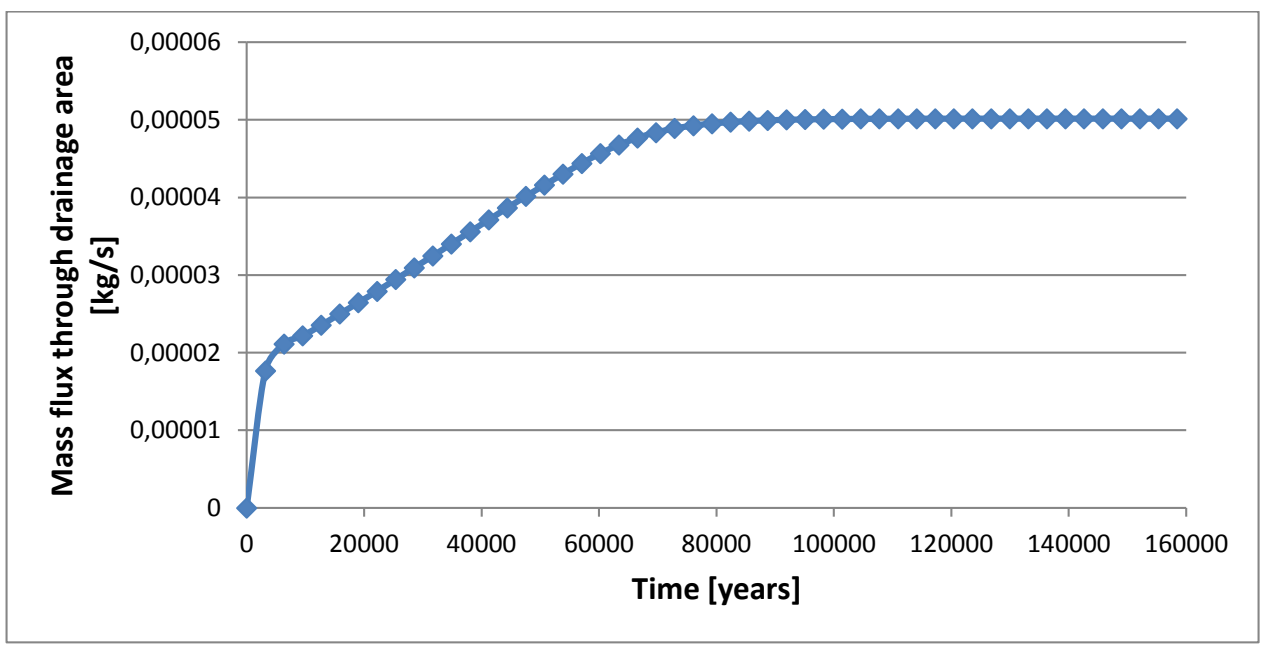

Figure 7. Test case B - mass flux through drainage area for constant source of concentration.

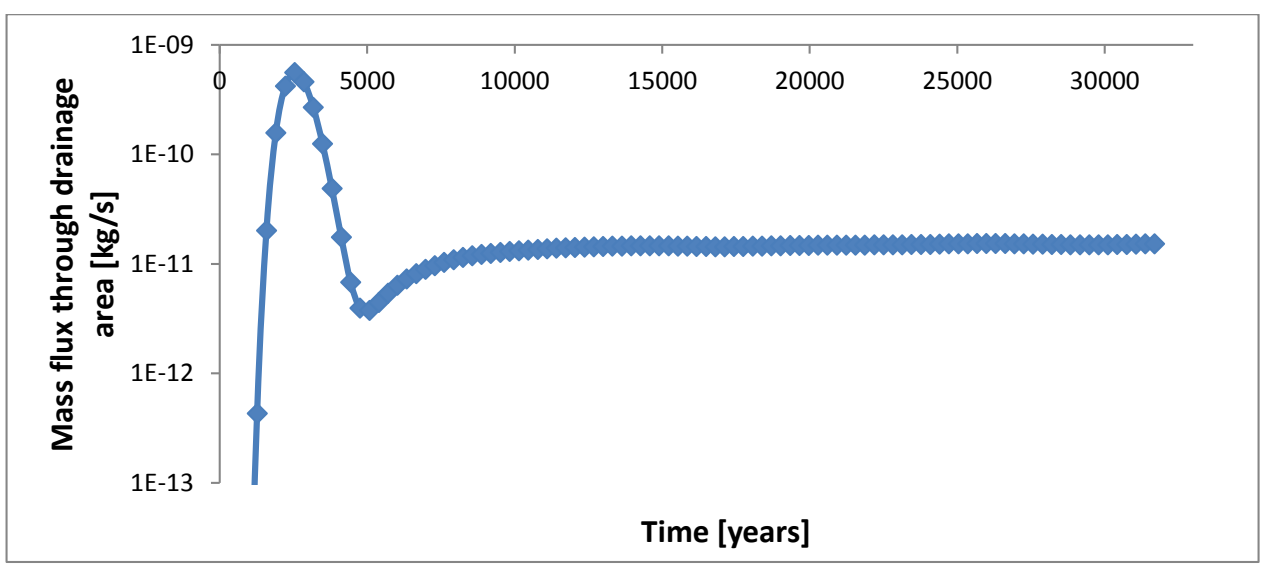

Figure 8 . Test case B - mass flux through drainage area for initial concentration.

\subsection{TEST CASE B}

The geometry of the second test case is shown in Figure 2 It is a $2 \mathrm{D}$ area with two fracture zones (their aperture is $1 \mathrm{~m}$ ). The surface of the area reaches altitudes from 250 to $350 \mathrm{~m}$. The subsurface layer of the high permeability rock is $100 \mathrm{~m}$ thick and its basis copies the surface. Parts of the surface that are within $50 \mathrm{~m}$ (in the direction of the $\mathrm{x}$ axis) from each minimum of the $\mathrm{z}$ value are marked as a drainage boundary (highlighted red in Figure 4); the rest of the surface is marked as an infiltration boundary.

Boundary conditions were prescribed so that the nature of the flow is given by the relief:

- On the drainage part, the Dirichlet boundary condition of zero pressure head.

- On the infiltration part, the Neumann boundary condition of constant flux equal to $1 E-9 \mathrm{~m} 3 / \mathrm{m} 2 / \mathrm{s}$.

- Homogeneous Neumann boundary condition on the rest of the boundaries.

Model parameters are summarized in Table 1

The flow and the solute transport were computed.

The simulation time for the transport was chosen so that the output concentration field is close to a stabilization. Based on the outputs of these simulations, the functional described above was computed and the transport path was generated for two different combinations of weights $\omega_{c}$ and $\omega_{v}$ (same as the ones described for the test case A). The results are shown in Figures 5 and 6 . The character of the transport path shown in Figure 6 may be variable in time, since it is influenced by the choice of time in which the concentration field is output and evaluated.

As for the case of the test case A, it is, again, possible to identify two different transport paths: the fastest one and the most abundant one. This is further documented by the graph in Figure 7. which depicts the dependence of mass flux through the drainage part of boundary on time for the case of constant source of concentration in the source element. The curve has two different gradient slopes. This can be interpreted as follows: the mass of the substance is transported to a boundary, through the fractures (the fastest path) first, and later it begins to reach a boundary via migration through a rock matrix (the most abundant path). Depending on a character of 
the source, any one of these transport paths may gain or lose significance. In the case of the constant source of concentration (Figure 7), the most abundant path seems to be the more significant one. In the case of the instantaneous release of concentration at the beginning of the simulation (prescribed as an initial condition in the source element) the fastest path seems to be the more significant one (Figure 8).

\section{Conclusions}

The alternative method based on a computation of the functional of velocity and concentration individually for each element of a mesh was proposed. Its applicability was verified on two test cases, which combined the EPM and the DFN modelling approaches (thus contained elements of different dimensions in a computational mesh). It was also shown that different choices of weights of the functional may be used to influence a character of the generated transport path, which might be useful in case we are interested not only in the fastest path through which the transported substance reaches a boundary in the shortest time but also in the most abundant path through which the biggest amount of substance is transported to a boundary in the given time. The most abundant transport path may be variable in time. The proposed method is able to account for this variability.

The vulnerability of this method is in its dependence on a discretization step, since it generates a transport path as a sequence of elements and not points in a continuous space. For very coarse meshes, the generated transport path may be significantly longer than it should be.

\section{ACKNOWLEDGEMENTS}

This publication is the result of the Radioactive Waste Repository Authority project „Research support for Safety Evaluation of Deep Geological Repository". This work was realized through the financial support of the Ministry of Education, Youth and Sports in the framework of the targeted support of the "National Programme for Sustainability I".

\section{REFERENCES}

[1] AHRENS, J., GEVECI, B. \& LAW, C., 2005. ParaView: An End-User Tool for Large-Data Visualization. Visualization Handbook, pp.717-731. DOI:10.1016/b978-012387582-2/50038-1

[2] Ayachit, Utkarsh, The ParaView Guide: A Parallel Visualization Application, Kitware, 2015, ISBN 978-1930934306

[3] Brezzi, F., \& Fortin, M. (2012). Mixed and hybrid finite element methods (Vol. 15). Springer Science \& Business Media.

[4] Březina, J. \& Hokr, M., 2011. Mixed-Hybrid Formulation of Multidimensional Fracture Flow. Lecture Notes in Computer Science, pp.125-132. DOI:10.1007/978-3-642-18466-6_14
[5] Březina, J.. Mortar-Like Mixed-Hybrid Methods for Elliptic Problems on Complex Geometries. In: D. Ševčovič A. Handlovičová, Z. Minarechová, editor, ALGORITMY 2012 - 19th Conference on Scientific Computing, September 9 - 14, 2012. Slovak University of Technology in Bratislava, Publishing House of STU, 2012.

[6] Clement, T.P. (1997). RT3D (Version 1.0): A Modular Computer Code for Simulating Reactive Multispecies Transport in 3-Dimensional Groundwater Systems. A Report for the U.S. Department of Energy. Richland, WA: Battelle Pacific Northwest Laboratory, p. 59.

[7] Darcy H., Les Fontaines publiques de lo ville de Dijon, Dalmont, Paris, 1856.

[8] Ferziger, J.H. \& Perić, M. Computational methods for fluid dynamics. Springer, 2002. DOI:10.1007/978-3-642-56026-2

[9] JACKSON, C.R. 2002. Steadystate particle tracking in the object-oriented regional groundwater model ZOOMQ3D. British Geological Survey Commissioned Report, CR/02/210C.

[10] Konikow, L.F., Goode, D.J., and Hornberger, G.Z. (1996). A Three-Dimensional Method-of-Characteristics Solute-Transport Model (MOC3D). U.S. Geological Survey Water Resources Investigation Report 96-4267. Reston, VA: U.S. Geological Survey.

[11] Pollock, D.W., 2016, User guide for MODPATH Version 7 - A particle-tracking model for MODFLOW: U.S. Geological Survey Open-File Report 2016-1086, 35 p., DOI:10.3133/ofr20161086

[12] Poteri, A., \& Laitinen, M. (1999). Site-to-canister scale flow and transport in Haestholmen, Kivetty, Olkiluoto and Romuvaara (POSIVA-99-15). Finland

[13] Roberts, J.E. \& Thomas, J.-M., Mixed and hybrid methods, in: Handbook of Numerical Analysis, Vol. 2, eds. P.G. Ciarlet and J.L. Lions (Elsevier Science B.V., Amsterdam, 1991) pp. 523-639.

[14] Singhal, B.B.S. \& Gupta, R.P., 2010. Applied Hydrogeology of Fractured Rocks. DOI:10.1007/978-90-481-8799-7

[15] Šístek, J., Březina, J. \& Sousedík, B., 2015. BDDC for mixed-hybrid formulation of flow in porous media with combined mesh dimensions. Numerical Linear Algebra with Applications, 22(6), pp.903-929. DOI:10.1002/nla.1991

[16] Whitaker, S. (1986). "Flow in porous media I: A theoretical derivation of Darcy's law". Transport in Porous Media. 1: 3-25. DOI:10.1007/BF01036523

[17] Willmann, M., G. W. Lanyon, P. Marschall, and W. Kinzelbach (2013), A new stochastic particle-tracking approach for fractured sedimentary formations, Water Resour. Res., 49, doi:10.1029/2012WR012191

[18] Zheng, C., Hill, M. C., Cao, G., \& Ma, R.. (2012). MT3DMS: Model Use, Calibration, and Validation. Transactions of the ASABE. American Society of Agricultural and Biological Engineers (ASABE). DOI:10.13031/2013.42263 